\title{
Anterolateral Approach in the Treatment of Femoroacetabular Impingement of the Hip
}

\author{
Hui Taek Kim, MD, Um Ji Kim, MD, Yoon Je Cho, MD \\ Department of Orthopedic Surgery, Pediatric Orthopedics and Biomedical Research Institute, \\ Pusan National University Hospital, Busan, Korea
}

\begin{abstract}
Background: Ganz surgical hip dislocation via a posterior (Kocher-Langenbeck) approach is a popular procedure in the management of femoroacetabular impingement (FAI). We report the results of surgery performed through an anterolateral (Watson-Jones) approach in the management of anterolateral FAl.

Methods: Twenty-one hips in 20 patients (mean age at the time of operation, 17.3 years) were treated surgically using an anterolateral approach: 12 hips with Legg-Calvé-Perthes disease, three septic hips, three hips with avascular necrosis (combined with slipped capital femoral epiphysis [SCFE], femoral neck fracture, and developmental dislocation of the hip), two hips with epiphyseal dysplasia, and one hip with SCFE. All patients had anterolateral FAl. Surgical hip dislocation was performed in four hips with trochanteric osteotomy. Combined osteotomies were for neck lengthening in 11 hips, varus or valgus osteotomy in the proximal femur in four hips, and pelvic osteotomy in four hips. Clinical results were evaluated using a modified Harris hip score (mHHS).

Results: Range of hip flexion and abduction showed statistically significant improvement after surgery; however, the improvement in mean mHHS was not statistically significant.

Conclusions: An anterolateral approach can be used as an alternative to a posterior approach in the management of anterolateral FAl with or without hip dislocation while safely preserving the blood supply to the femoral head and allowing simultaneous procedures in the proximal femur.

Keywords: Femoroacetabular impingement, Hip dislocation
\end{abstract}

Femoroacetabular impingement (FAI) often follows hip disorders in children and adolescents. Management of FAI is important not only for improvement of the symptoms but also for prevention of osteoarthritis. Although conservative treatment is effective in managing mild cases of FAI, more serious cases require surgery even though this involves a delayed return to activity and a larger scar. Arthroscopy has become a widely used technique in many joint opera-

Received January 2, 2019; Revised March 11, 2019; Accepted March 25, 2019

Correspondence to: Hui Taek Kim, MD

Department of Orthopedic Surgery, Pediatric Orthopedics and Biomedical Research Institute, Pusan National University Hospital, 179 Gudeok-ro, Seo-gu, Busan 49241, Korea

Tel: +82-51-240-7248, Fax: +82-51-247-8395

E-mail: kimht@pusan.ac.kr tions, but it requires a steep learning curve for FAI.

Ganz surgical hip dislocation by a posterior approach, ${ }^{1)}$ using the Gibson ${ }^{2)}$ or Kocher-Langenbeck incision, ${ }^{3)}$ is a popular procedure in the management of FAI with preservation of the blood supply to the femoral head. ${ }^{4)}$ However, this technique may be too aggressive and invasive for some cases. The anterolateral (WatsonJones) approach ${ }^{5)}$ in the supine position is well known and less invasive for mild to moderate degrees of anterolateral FAI. This allows preservation of both extra- and intracapsular segments of the deep branch of the medial femoral circumflex artery (MFCA $)^{4)}$ without approaching the posterior aspect of the hip joint. Combined osteotomy in the proximal femur can be done using the same approach, and surgical hip dislocation with trochanteric osteotomy is also possible. This paper reports our experience of using the anterolateral (Watson-Jones) approach to manage FAI 


\section{Kim et al. Femoroacetabular Impingement}

Clinics in Orthopedic Surgery • Vol. 11, No. 3, 2019 • www.ecios.org

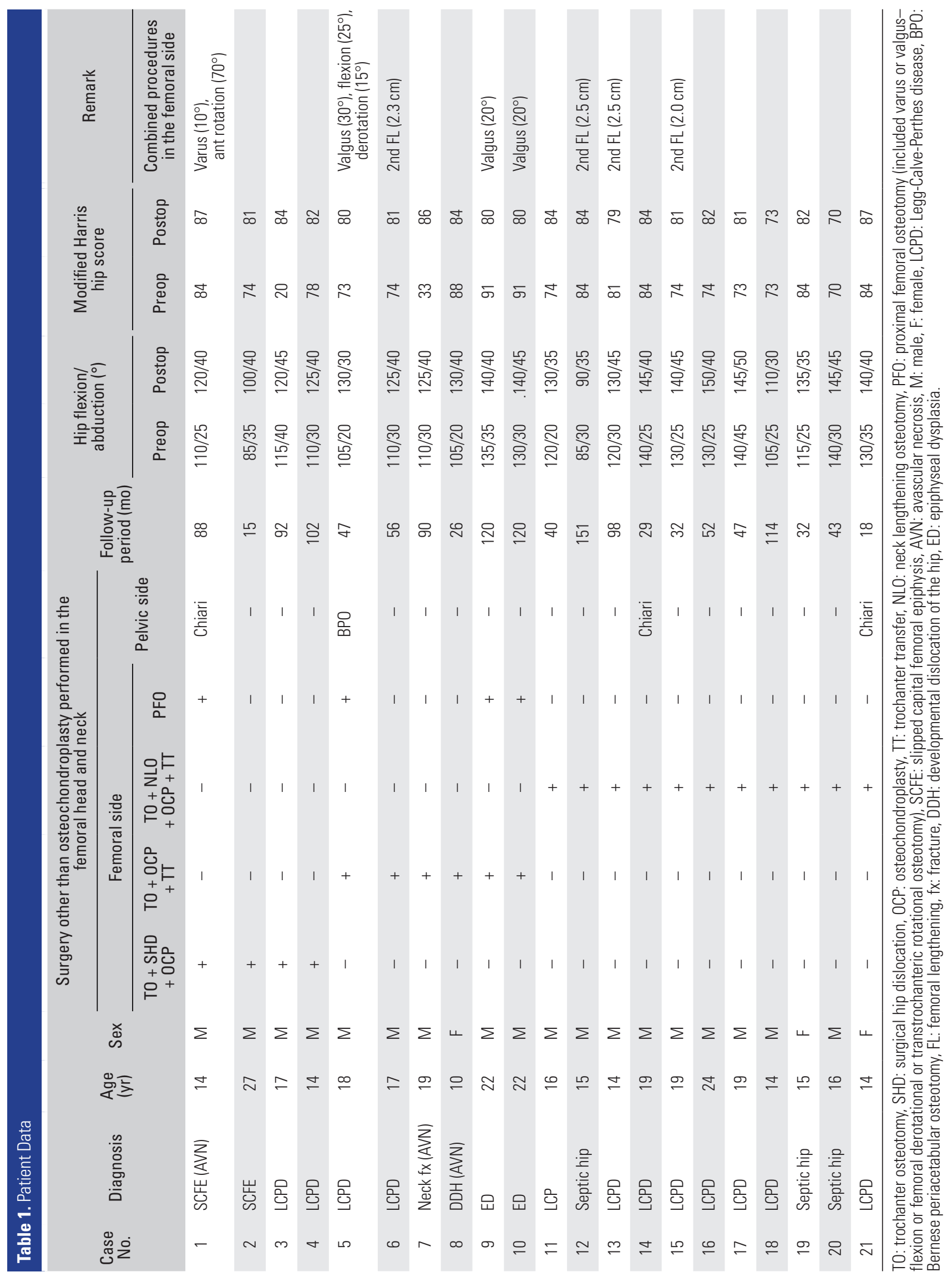


in various hip disorders.

\section{METHODS}

Twenty-one hips in 20 patients with FAI were treated using an anterolateral approach (Table 1). Informed consent for participation in the study was obtained from all patients or parents and the study was approved by the Instituional Review Board of Pusan National University Hospital (IRB No. H-1906-031-080). There were 17 men and three women. Mean age at the time of operation was 17.3 years (range, 10 to 27). Patients were followed up for an average of 5.6 years (range, 1.3 to 12.6). Primary diagnosis included 12 hips with Legg-Calvé-Perthes disease, three with septic hip, three with avascular necrosis with slipped capital femoral epiphysis (SCFE), femoral neck fracture, and developmental dislocation of the hip, two with epiphyseal dysplasia, and one with SCFE. Combined procedures included neck lengthening osteotomy ${ }^{6}$ in the proximal femur (11 cases), trochanteric osteotomy for surgical hip dislocation (four cases), varus or valgus osteotomy in the proximal femur (four cases), and pelvic osteotomy (four cases; one Bernese periacetabular and three Chiari). Our indication for the anterolateral approach was impingement located on the anterior or anterolateral aspect of the femoral head and neck.

\section{Surgical Procedure}

Each patient was prepared and draped in the supine position with a bump under the buttock on the operative side and supported by a kidney rest on the opposite side. Fluoroscopic evaluation was performed throughout the operation facilitated with a radiolucent operating table. The skin incision was started $2.5 \mathrm{~cm}$ posterior and distal to the anterior superior iliac spine, and then it was curved distally and posteriorly to the greater trochanter and extended $5 \mathrm{~cm}$ distal to the greater trochanter along the shaft of the femur. ${ }^{5}$ The interval between the tensor fascia latae and gluteus medius was then located. Blunt dissection was performed carefully to identify the joint capsule, avoiding injury to the inferior branch of the superior gluteal nerve that innervates the tensor fasciae latae muscle and anterior gluteus medius. The vastus lateralis and vastus ridge were then identified, and electrocautery was used to retract the muscle proximally $1-2 \mathrm{~cm}$ from its origin. Blunt dissection was then continued to expose the capsule.

\section{Capsulotomy without trochanteric osteotomy}

The capsule was incised longitudinally along the anterosuperior surface of the femoral neck and a T-shaped cut was made along the acetabular rim. With this approach, the femoral head blood supply could be preserved along with the anterior inferior capsule. Subsynovial retinacular vessels from the deep branch of the MFCA, which enter the posterolateral aspect of the femoral head, ${ }^{4)}$ were thus safely preserved (Fig. 1). The hip joint was then exposed to allow inspection of the preoperatively evaluated impingement site. The osteochondroplasty in the anterolateral aspect of the femoral head and neck was performed while the leg was manipulated simultaneously. If the operative field was expanded over the anterolateral area of the hip joint, detachment of the anterior fibers of the gluteus medius tendon from the trochanter was performed for better visualization, or the trochanter was osteotomized to allow for hip dislocation.

\section{Trochanteric osteotomy for hip dislocation}

The trochanter was osteotomized from a posterior to anterior direction because the deep branch of the medial femoral circumflex artery (MFCA) runs along the trochanteric crest in close proximity to the osteotomy site (Fig. 1). Careful identification and exposure of the posterior border of the greater trochanter was necessary before performing the osteotomy. Firm reattachment of the greater trochanter

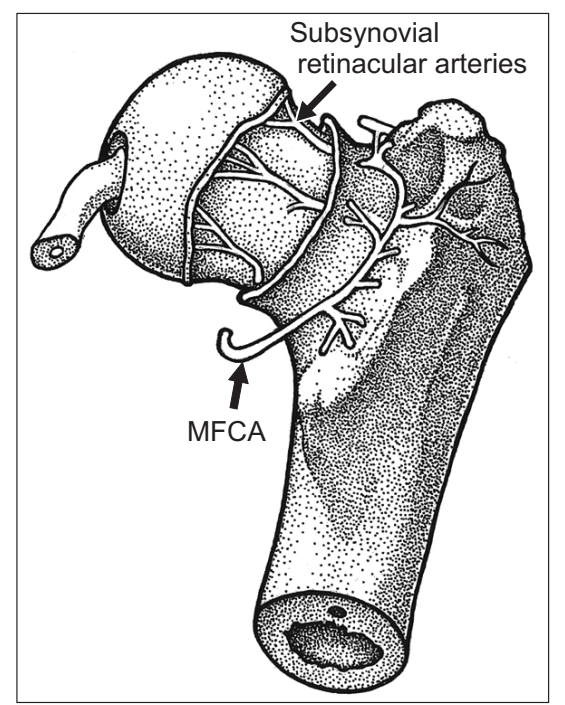

Fig. 1. Arteries of the femoral head and neck viewed from a posterior aspect (surrounding muscles are removed). Subsynovial retinacular arteries enter the capsule from the medial femoral circumflex artery (MFCA). Subperiosteal stripping from the lateral aspect of the femur to the medial side (proximally from the level of the lesser trochanter) and a careful cut of the short external rotators along the bony cortex prevent vascular damage during hip dislocation and simultaneous proximal femoral osteotomy. 
Kim et al. Femoroacetabular Impingement

Clinics in Orthopedic Surgery • Vol. 11, No. 3, $2019 \bullet$ www.ecios.org

was done using $4.5-\mathrm{mm}$ cannulated screws after the operation.

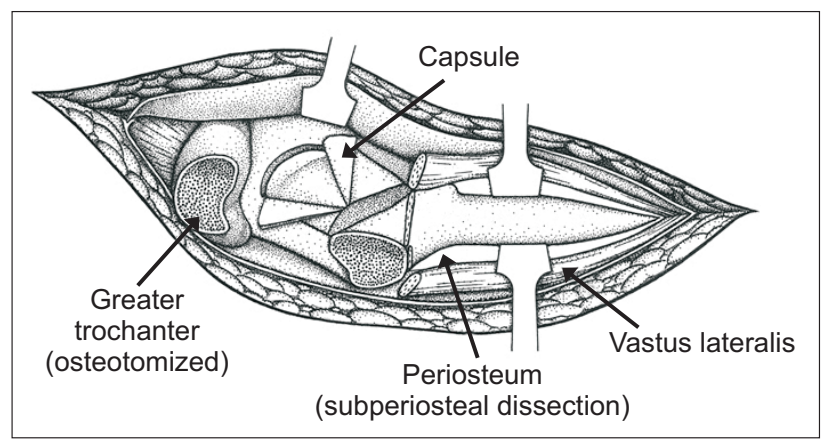

Fig. 2. Illustration of the hip joint and femur before dislocation of the femoral head and osteotomy in the proximal femur showing an osteotomized greater trochanter, opened capsule, longitudinally split vastus lateralis, and subperiosteal dissection to protect the medial femoral circumflex artery.
Subperiosteal flap dissection in the posterior half of the proximal femur for hip dislocation

When hip dislocation with trochanteric osteotomy was required for wider exposure of the femoral head or simultaneous procedures in the proximal femur were necessary, we performed subperiosteal flap dissection in the posterior half of the proximal femur. For this, the vastus lateralis muscle and periosteum were divided longitudinally on the lateral side of the femur from the vastus ridge. Posterior subperiosteal flap dissection was performed proximally starting from the lateral side to the medial side at the level of the lesser trochanter (Fig. 2)-where the deep branch of the MFCA runs dorsally between the iliopsoas and the pectineus muscle and continues proximal to the lesser trochanter at the base of the femoral neck (Fig. 1) along the inferior border of the obturator externus muscleto the posterosuperior aspect of the neck of the femurwhere the subsynovial retinacular vessels enter the femoral head. Subperiosteal flap dissection protected the MFCA while we were performing procedures in the proximal fe-
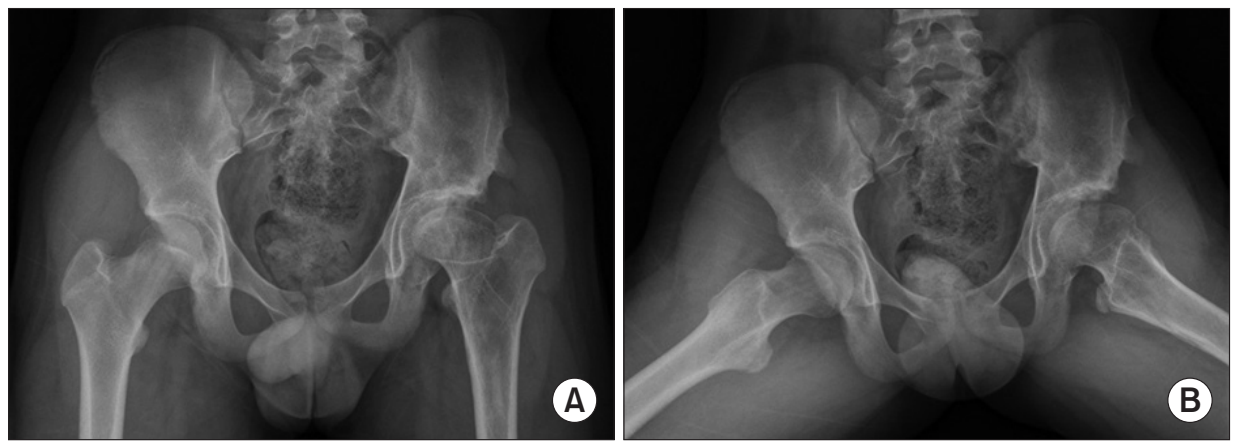

Fig. 3. Anteroposterior (A) and frog-leg lateral (B) views of a 14-year-old boy with Legg-Calvé-Perthes disease in the left hip show a short femoral neck, overgrown greater trochanter, and a 2-cm leg length discrepancy. At age 9 years, he underwent a valgus-flexion femoral osteotomy and then Chiari pelvic osteotomy due to the laterally uncovered femoral head. Clinically he showed limping and limited range of motion, especially in abduction.
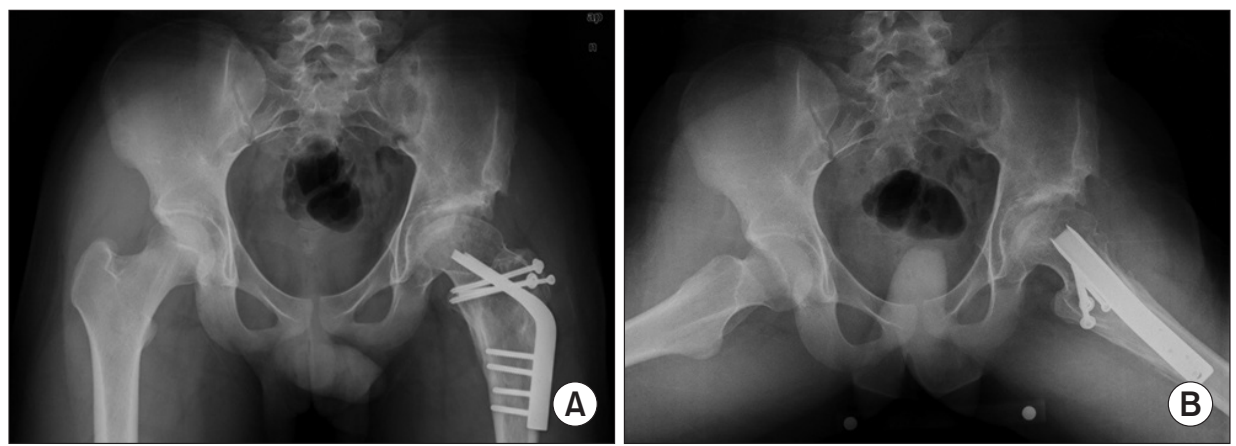

Fig. 4. A patient underwent a neck lengthening osteotomy with lateral and distal transfer of the greater trochanter performed through an anterolateral approach. Simultaneous osteochondroplasty of the anterolateral aspect of the femoral head and neck was also performed through the same approach. Anteroposterior (A) and frog-leg lateral (B) views taken 3 years after surgery (at age 17) show improved biomechanical anatomy of the hip. 
mur. The short external rotators were also carefully cut by electrocautery (at low voltage) along the bony cortex for hip dislocation. Thorough understanding of the anatomy of the MFCA and retinacular arteries was crucial to avoid damaging these vessels. The external rotators were then repaired after the operation by suturing to the remaining soft tissues or to the drilled bone tunnels or by suture anchors.

Capsulotomy for hip dislocation after trochanteric osteotomy When surgical hip dislocation was necessary, we used a Zshaped capsulotomy as follows: ${ }^{1)}$ The superior limb of the capsulotomy was located along the posterior acetabular rim, and the inferior limb was located at the level of the anteromedial femoral neck. Capsulotomy of the longitudinal limb was done in line with the femoral neck along its anterolateral surface, connecting the superior and the anterior limbs to complete the capsulotomy. Thus, the retinacular vessels entering the capsule at the inferior area of the superolateral neck were protected.

\section{Combined procedures in the proximal femur}

Simultaneous neck lengthening osteotomy and varus/ valgus/flexion osteotomy in the proximal femur were then performed following the above treatment for FAI (Figs. 3-5).

\section{Clinical Evaluation}

The ranges of hip flexion and abduction were measured with a goniometer while the patient's hip was moved passively by the examiner. The end points of each motion were noted when pain occurred. Clinical results were evaluated using a modified Harris hip score (mHHS).

\section{Statistics}

Paired $t$-tests were used to compare pre- and postoperative mHHS and flexion and abduction ranges.

\section{RESULTS}

There was a statistically significant improvement in the range of hip flexion and abduction after surgery; but not in clinical outcomes (Table 2). Range of hip flexion improved from a mean of $117.6^{\circ} \pm 15.8^{\circ}$ to $129.8^{\circ} \pm 15.1^{\circ}$; that of abduction improved from a mean of $29.3^{\circ} \pm 6.4^{\circ}$ to $39.5^{\circ}$ $\pm 5.2^{\circ}$. Mean mHHS improved from $74.3 \pm 16.8$ points to $81.5 \pm 3.9$ points. There were no postoperative complications.

Table 2. Preoperative and Postoperative Ranges of Motion and Harris Hip Score for 21 Hips Treated for FAl

\begin{tabular}{lc}
\multicolumn{1}{c}{ Variable } & Mean \pm SD (range) \\
Hip Flexion $\left(^{\circ}\right)$ & $117.6 \pm 15.8(85-140)$ \\
Preoperative & $129.8 \pm 15.1(90-150)$ \\
Postoperative & 0.000 \\
${ }^{*} p$-value & \\
\hline Hip abduction $\left(^{\circ}\right)$ & $29.3 \pm 6.4(20-45)$ \\
\hline Preoperative & $39.5 \pm 5.2(30-50)$ \\
\hline Postoperative & 0.026 \\
\hline${ }^{*} p$-value & \\
\hline Modified Harris hip score & $74.3 \pm 16.8(20-91)$ \\
\hline Preoperative & $81.5 \pm 3.9(70-87)$ \\
\hline Postoperative & 0.076 \\
\hline$p$-value & \\
\hline
\end{tabular}

FAl: femoroacetabular impingement, SD: standard deviation.

*Paired $t$-test showed a significant improvement of flexion and abduction after surgery $(p<0.05)$.
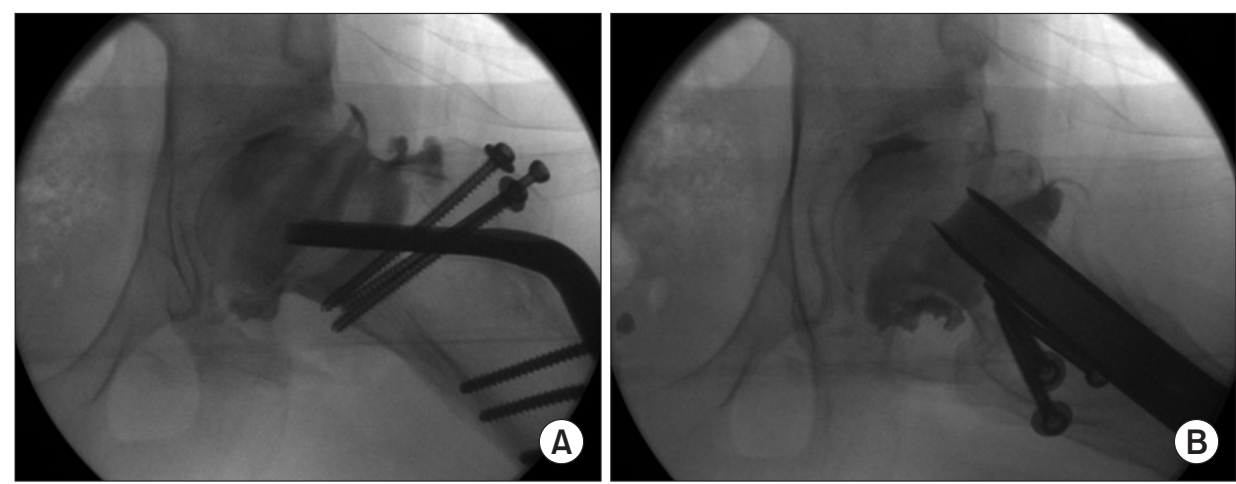

Fig. 5. Abduction (A) and lateral (B) arthrographs show improved sphericity of the femoral head. The hip range of motion improved by $10^{\circ}$ in flexion and $15^{\circ}$ in abduction. 


\section{DISCUSSION}

Selection of the best approach for surgical treatment of FAI must be based on the cause and location of the impingement, as determined by careful use of the available imaging technology. Many cases of FAI in various hip disorders require open surgery because of poor visualization of both the inferoposterior labrum and central chondral lesions in the mini-open osteochondroplasty and because of the steep learning curve for hip arthroscopy. In addition, simultaneous reorientation of the proximal femur with a valgus-flexion intertrochanteric osteotomy is also required to reduce FAI in cases where the femoral neck is short or varus deformed. Relative neck lengthening procedures ${ }^{6}$ with trochanteric advancement present another possibility of increasing clearance.

The anterolateral approach $^{5)}$ is indicated when the landmarks requiring resection are located on the anterolateral aspect of the femoral head and neck. This is a moderately invasive approach as opposed to the mini-open, ${ }^{7-10)}$ arthroscopy, ${ }^{11-16)}$ and surgical hip dislocation with trochanteric osteotomy through a posterior approach. ${ }^{17-25)} \mathrm{In}$ the adult, the vascular supply to the femoral head comes through the retinacular arteries from the deep branch of the MFCA..$^{1,4,26,27)}$ Both the anterolateral and posterior approaches risk damaging the deep branch and/or the extracapsular portion of the MFCA, when its exact course to the femoral head is not precisely known. Minimizing the risk of damage to the vascular supply of the femoral head will always be essential in both approaches.

If an anterolateral approach can achieve the same results as the posterior approach in the treatment of FAI, we believe the former is safer, especially for the surgeon who is relatively inexperienced regarding the anatomy of the MFCA and its branches to the femoral head and neck. The majority of patients with FAI have lesions located in either the anterosuperior or anteroinferior quadrants $(86 \%$ and $94 \%$ in two series), ${ }^{28,29)}$ further justifying an anterolateral approach. Generally, a supine position in the anterolateral approach reduces the time required for draping the pa- tient and provides more stability when the hip is moved to check for impingement and for performing other femoral procedures. Anterior capsulotomy performed proximally near the acetabular rim with preservation of the anterior inferior capsule can preserve the blood supply from the MFCA.

Our subperiosteal flap dissection from the level of the lesser trochanter to the posterosuperior aspect of the femoral neck protects the MFCA from damage during more extensive surgery (when hip dislocation and proximal femoral osteotomy are required). When it is required to access the anterolateral and posterior aspect of the femoral head, or even the acetabulum, a trochanteric osteotomy can be performed to expose the whole capsule in the anterolateral approach. The short external rotators are carefully cut to facilitate hip dislocation with a Zshaped capsulotomy, while the retinacular vessels entering the capsule at the inferior area of the superolateral neck are carefully protected as in hip dislocation performed through a posterior approach.

In conclusion, when a mild or moderate anterolateral bump accompanies FAI, an anterolateral approach can be considered as an alternative to the Ganz-type surgical hip dislocation through a posterior approach. We obtained satisfactory outcomes by using an anterolateral approach with or without hip dislocation. This approach allows inspection and treatment of the preoperatively evaluated anterolateral impingement site. Combined procedures in the proximal femur can be performed through the same approach.

\section{CONFLICT OF INTEREST}

No potential conflict of interest relevant to this article was reported.

\section{ACKNOWLEDGEMENTS}

This work was supported by a 2-Year Research Grant of Pusan National University.

\section{REFERENCES}

1. Ganz R, Gill TJ, Gautier E, Ganz K, Krugel N, Berlemann U. Surgical dislocation of the adult hip a technique with full access to the femoral head and acetabulum without the risk of avascular necrosis. J Bone Joint Surg Br. 2001;83(8):1119-24.

2. Gibson A. Posterior exposure of the hip joint. J Bone Joint Surg Br. 1950;32(2):183-6.
3. Letournel E, Judet R. Fractures of the Acetabulum. 2nd ed. Berlin: Springer Verlag; 1993. 364-9.

4. Gautier E, Ganz K, Krugel N, Gill T, Ganz R. Anatomy of the medial femoral circumflex artery and its surgical implications. J Bone Joint Surg Br. 2000;82(5):679-83.

5. Watson-Jones, R. Fractures of the neck of the femur. Br J 
Kim et al. Femoroacetabular Impingement

Clinics in Orthopedic Surgery • Vol. 11, No. 3, $2019 \bullet$ www.ecios.org

Surg. 1936;23(92):787-808

6. Buess P, Morscher E. Osteotomy to lengthen the femur neck with distal adjustment of the trochanter major in coxa vara after hip dislocation. Orthopade. 1988;17(6):485-90.

7. Ribas M, Ledesma R, Cardenas C, Marin-Pena O, Toro J, Caceres E. Clinical results after anterior mini-open approach for femoroacetabular impingement in early degenerative stage. Hip Int. 2010;20 Suppl 7:S36-42.

8. Ng VY, Arora N, Best TM, Pan X, Ellis TJ. Efficacy of surgery for femoroacetabular impingement: a systematic review. Am J Sports Med. 2010;38(11):2337-45.

9. Parvizi J, Huang R, Diaz-Ledezma C, Og B. Mini-open femoroacetabular osteoplasty: how do these patients do? J Arthroplasty. 2012;27(8 Suppl):122-5.

10. Cohen SB, Huang R, Ciccotti MG, Dodson CC, Parvizi J. Treatment of femoroacetabular impingement in athletes using a mini-direct anterior approach. Am J Sports Med. 2012;40(7):1620-7.

11. Philippon M, Schenker M, Briggs K, Kuppersmith D. Femoroacetabular impingement in 45 professional athletes: associated pathologies and return to sport following arthroscopic decompression. Knee Surg Sports Traumatol Arthrosc. 2007;15(7):908-14.

12. Byrd JW, Jones KS. Arthroscopic management of femoroacetabular impingement: minimum 2-year follow-up. Arthroscopy. 2011;27(10):1379-88.

13. Bedi A, Galano G, Walsh C, Kelly BT. Capsular management during hip arthroscopy: from femoroacetabular impingement to instability. Arthroscopy. 2011;27(12):1720-31.

14. Nho SJ, Magennis EM, Singh CK, Kelly BT. Outcomes after the arthroscopic treatment of femoroacetabular impingement in a mixed group of high-level athletes. Am J Sports Med. 2011;39 Suppl:14S-19S.

15. Alradwan H, Philippon MJ, Farrokhyar F, et al. Return to preinjury activity levels after surgical management of femoroacetabular impingement in athletes. Arthroscopy. 2012;28(10):1567-76.

16. Malviya A, Stafford GH, Villar RN. Impact of arthroscopy of the hip for femoroacetabular impingement on quality of life at a mean follow-up of 3.2 years. J Bone Joint Surg Br. 2012;94(4):466-70.

17. Beck M, Leunig M, Parvizi J, Boutier V, Wyss D, Ganz R. Anterior femoroacetabular impingement: part II. Midterm results of surgical treatment. Clin Orthop Relat Res.
2004;(418):67-73.

18. Murphy S, Tannast M, Kim YJ, Buly R, Millis MB. Debridement of the adult hip for femoroacetabular impingement: indications and preliminary clinical results. Clin Orthop Relat Res. 2004;(429):178-81.

19. Beaule PE, Le Duff MJ, Zaragoza E. Quality of life following femoral head-neck osteochondroplasty for femoroacetabular impingement. J Bone Joint Surg Am. 2007;89(4):773-9.

20. Yun HH, Shon WY, Yun JY. Treatment of femoroacetabular impingement with surgical dislocation. Clin Orthop Surg. 2009;1(3):146-54.

21. Kempthorne JT, Armour PC, Rietveld JA, Hooper GJ. Surgical dislocation of the hip and the management of femoroacetabular impingement: results of the Christchurch experience. ANZ J Surg. 2011;81(6):446-50.

22. Naal FD, Miozzari HH, Wyss TF, Notzli HP. Surgical hip dislocation for the treatment of femoroacetabular impingement in high-level athletes. Am J Sports Med. 2011;39(3):544-50.

23. Papalia R, Del Buono A, Franceschi F, Marinozzi A, Maffulli N, Denaro V. Femoroacetabular impingement syndrome management: arthroscopy or open surgery? Int Orthop. 2012;36(5):903-14.

24. Sink EL, Fabricant PD, Pan Z, Dayton MR, Novais E. Results of treatment of femoroacetabular impingement in adolescents with a surgical hip dislocation approach. Clin Orthop Relat Res. 2013;471(8):2563-9.

25. Shin SJ, Kwak HS, Cho TJ, et al. Application of Ganz surgical hip dislocation approach in pediatric hip diseases. Clin Orthop Surg. 2009;1(3):132-7.

26. Trueta J, Harrison MH. The normal vascular anatomy of the femoral head in adult man. J Bone Joint Surg Br. 1953;35(3):442-61.

27. Sevitt S, Thompson RG. The distribution and anastomoses of arteries supplying the head and neck of the femur. J Bone Joint Surg Br. 1965;47(3):560-73.

28. McCarthy JC, Noble PC, Schuck MR, Wright J, Lee J. The Otto E. Aufranc Award: the role of labral lesions to development of early degenerative hip disease. Clin Orthop Relat Res. 2001;(393):25-37.

29. Mintz DN, Hooper T, Connell D, Buly R, Padgett DE, Potter HG. Magnetic resonance imaging of the hip: detection of labral and chondral abnormalities using noncontrast imaging. Arthroscopy. 2005;21(4):385-93. 